\title{
An Efficient Signal Reconstruction Algorithm for Stepped Frequency MIMO-SAR in the Spotlight and Sliding Spotlight Modes
}

\author{
Jiajia Zhang, Guangcai Sun, Mengdao Xing, Zheng Bao, and Fang Zhou \\ Key Laboratory for Radar Signal Processing, Xidian University, Xian 710071, China \\ Correspondence should be addressed to Jiajia Zhang; zjjreal@126.com
}

Received 25 January 2014; Revised 13 June 2014; Accepted 16 June 2014; Published 26 June 2014

Academic Editor: Mathini Sellathurai

Copyright (c) 2014 Jiajia Zhang et al. This is an open access article distributed under the Creative Commons Attribution License, which permits unrestricted use, distribution, and reproduction in any medium, provided the original work is properly cited.

Multiple-input multiple-output (MIMO) synthetic aperture radar (SAR) using stepped frequency (SF) waveforms enables a high two-dimensional (2D) resolution with wider imaging swath at relatively low cost. However, only the stripmap mode has been discussed for SF MIMO-SAR. This paper presents an efficient algorithm to reconstruct the signal of SF MIMO-SAR in the spotlight and sliding spotlight modes, which includes Doppler ambiguity resolving algorithm based on subaperture division and an improved frequency-domain bandwidth synthesis (FBS) method. Both simulated and constructed data are used to validate the effectiveness of the proposed algorithm.

\section{Introduction}

According to the minimum antenna area constraint, high resolution and wide swath (HRWS) pose contradicting requirements on synthetic aperture radar (SAR) system design [1]. This system-inherent limitation can be overcome by singleinput multiple-output (SIMO) SAR system [2], which has been extended to multiple SAR modes, such as spotlight and sliding spotlight, to fulfill the different requirements of spatial resolution and coverage in future SAR missions [3-5]. In high-resolution SAR, wide bandwidth signals are needed to obtain a high range resolution. However, the direct transmission of a wide bandwidth signal may lead to a high cost of the hardware [6-8]. A solution to this problem is the transmission of stepped frequency (SF) waveforms in combination with bandwidth synthesis technologies [6-8].

The SF multiple-input multiple-output (MIMO)-SAR, which is defined as the MIMO-SAR system transmitting and receiving a class of SF subband signals simultaneously by multiple azimuth channels, respectively, combines the advantages of the SIMO system and SF waveforms and enables HRWS imaging at relatively low cost $[9,10]$. However, only the stripmap mode SF MIMO-SAR has been discussed.
In the spotlight and sliding spotlight modes, the steering of the antenna beams leads to an increase of the azimuth bandwidth and thus raises difficulties for both azimuth reconstruction and bandwidth synthesis. Furthermore, the HRWS leads to a great amount of data, which may cause high computational burden, so an efficient signal reconstruction algorithm is required.

In this paper, an efficient algorithm is presented to reconstruct SF MIMO-SAR signal for the spotlight and sliding spotlight modes. Firstly, a Doppler ambiguity resolving algorithm based on the azimuth subaperture division is presented for azimuth reconstruction of each SF subband signal. Then, an improved frequency-domain bandwidth synthesis method without upsampling in range is proposed to efficiently synthesize the subband signals.

This paper is organized as follows. In Section 2, the signal model of SF MIMO-SAR is introduced. In Section 3, the Doppler ambiguity resolving algorithm based on subaperture division is described. An improved FBS method without upsampling in range is proposed and the flowchart of the signal reconstruction algorithm is shown in Section 4. In Section 5, simulation and real data processing results are presented. Finally, the conclusion is presented in Section 6. 


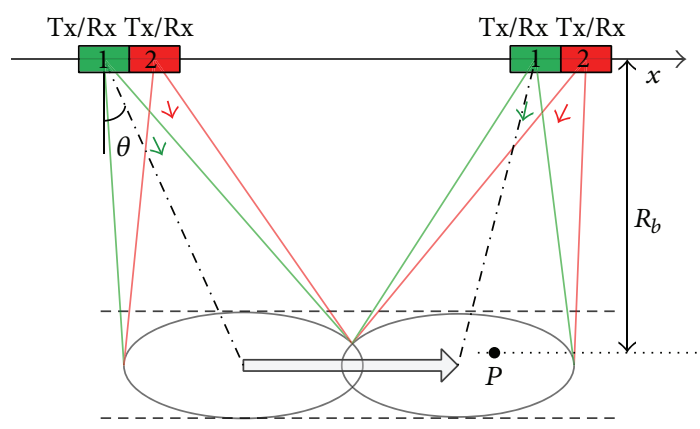

FIGURE 1: Imaging geometry of SF MIMO-SAR (sliding spotlight).

\section{Signal Model}

Consider a SF MIMO-SAR system with $N$ transmit/receive $(\mathrm{Tx} / \mathrm{Rx})$ channels in azimuth by which a class of SF subband signals are transmitted at the same time, respectively. The carrier frequencies of this class of subband signals increase from subband to subband by a fixed frequency step $\Delta f_{c}$, and then the center frequency of the subband $n$ can be represented by

$$
f_{n}=f_{c}+\Delta f_{c}\left[n-\frac{(N+1)}{2}\right]=f_{c}+\Delta f_{c, n}
$$

where $f_{c}$ is the center frequency of the whole synthesized bandwidth signal and $\Delta f_{c, n}$ denotes the center frequency interval between the $n$th subband signal and the whole bandwidth signal.

Figure 1 shows the imaging geometry of sliding spotlight SF MIMO-SAR with two channels as an example. The radar moves along the $x$-axis with a constant velocity $v$, and the main beams of $N$ transmit/receive (Tx/Rx) channels in azimuth are steered from forward to backward, respectively, pointing to the rotation center of the corresponding mode. The echoes are received by all the $N$ channels simultaneously, so $N$ channels data are achieved for each subband signal. If the azimuth multichannel samples of each subband signal do not coincide, the PRF will be improved $N$ times after azimuth reconstruction, so the equivalent PRF after azimuth reconstruction is $N \cdot \mathrm{PRF}$. In order to ensure relative wide range coverage, the $\mathrm{PRF}$ is set to ensure the equivalent PRF after azimuth reconstruction to be lower than the total Doppler bandwidth $B_{\text {azi }}$ and slightly greater than the instantaneous Doppler bandwidth $B_{\text {ins }}[4,5]$.

Suppose that $X_{n}$ and $X_{m}$, respectively, denote the relative positions in azimuth of the $n$th transmit channel (Tx $n$ ) and the $m$ th $(m=1,2, \ldots, N)$ receive channel $(\mathrm{Rx} m)$. The multichannel data of each subband signal can be converted into equivalent self-transmitting and self-receiving data corresponding to the effective phase centers (EPCs) by compensating the phase errors [8]. The compensating function for the subband $n$ signal received by $\mathrm{Rx} m$ is denoted by $H_{1, n}(m)$. After the compensation of the phase errors,
$N$ EPCs signal can be achieved for subband $n$ signal, whose azimuth coordinate can be given as

$$
X_{n m}^{\prime}=\frac{\left(X_{n}+X_{m}\right)}{2} \text {. }
$$

The EPC corresponding to Txl and Rxl is defined as the reference EPC, whose azimuth coordinate is set to be zero. It is shown in (2) that, due to different positions of transmit channels, the EPCs of different subband signals corresponding to a same $\mathrm{Rx}$ channel are of different azimuth coordinates. These geometry differences between subband signals should be removed for the bandwidth synthesis.

After range compression, the signal of a point target $P\left(X, R_{b}\right)$ transmitted by $\mathrm{Tx} n$ and received by $\mathrm{Rx} m$ can be expressed as

$$
\begin{aligned}
s_{1, n}\left(t, t_{a}, m\right)= & w_{r}\left(t-\frac{2}{c} R_{n m}\left(t_{a}\right)\right) \\
& \times w_{\text {azi }}\left(t_{a}+\frac{X_{n m}^{\prime}-X}{v}\right) \\
& \times \exp \left(-j \frac{4 \pi f_{n}}{c} R_{n m}\left(t_{a}\right)\right),
\end{aligned}
$$

where $R_{n m}\left(t_{a}\right)=\sqrt{R_{b}^{2}+\left(v t_{a}+X_{n m}^{\prime}-X\right)^{2}}$ is the slant range from point $P$ to the EPC corresponding to Tx $n$ and $\mathrm{Rx} m, c$ is the speed of light, $t$ is the fast time, $t_{a}$ is the slow time, and $w_{r}(\cdot)$ and $w_{\text {azi }}(\cdot)$ denote the range window function and the azimuth window function, respectively.

\section{Doppler Ambiguity Resolving Based on Subaperture Division}

In the spotlight and sliding spotlight modes, the time-variant squint angle inevitably leads to an extension of the azimuth spectrum $[4,5]$. The time-frequency distribution (TFD) of the sliding spotlight mode is shown in Figure 2(a). The azimuth reconstruction processing based on the subaperture division for the sliding spotlight SIMO SAR [4] can be extended to SF MIMO-SAR in the spotlight and sliding spotlight modes to resolve Doppler ambiguity of each subband signal. The subaperture division of subband signals should ensure that the processed Doppler bandwidth of the whole bandwidth signal is smaller than the equivalent PRF. Since the whole bandwidth is very wide to obtain a high resolution, the influence of range frequency on the processed Doppler bandwidth should be taken into account, which is different from the case in [4].

The beam central frequency varying with slow time $t_{a}$ and range frequency can be expressed as

$$
f_{d c}(f)=\frac{2 v \sin \left[\theta\left(t_{a}\right)\right]}{c} f
$$

where $f \in\left[f_{c}-N \Delta f_{c} / 2, f_{c}+N \Delta f_{c} / 2\right]$ is the range frequency, which covers all the subbands and $\theta$ denotes the timevariant azimuth squint angle. From Figure 2(b), the processed 


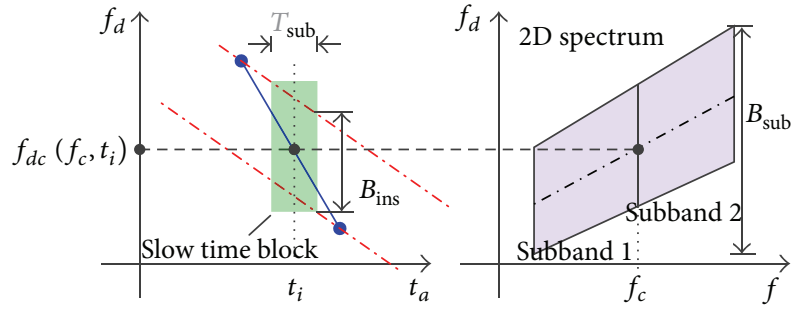

FIGURE 2: The subaperture division. (a) The TFD of sliding spotlight. (b) $2 \mathrm{D}$ spectrum of the whole bandwidth signal.

azimuth bandwidth $B_{\text {sub }}$ of the whole bandwidth signal can be approximately expressed as

$$
\begin{aligned}
B_{\text {sub }} \approx & B_{\text {ins }}+k_{\text {rot }} T_{\text {sub }}+f_{d c, i}\left(f_{c}+\frac{N \Delta f_{c}}{2}\right) \\
& -f_{d c, i}\left(f_{c}-\frac{N \Delta f_{c}}{2}\right),
\end{aligned}
$$

where $k_{\text {rot }}=2 v^{2} f_{c} / c R_{\text {rot }}$ denotes the slope rate of the variation of the beam central Doppler frequency, $R_{\text {rot }}$ is the rotational distance, and $f_{d c, i}$ denotes the Doppler center of the $i$ th subaperture, which varies with the range frequency. To reconstruct the Doppler spectrum of the whole bandwidth signal, the duration of each slow time block $T_{\text {sub }}$ is chosen to ensure that the processed bandwidth $B_{\text {sub }}$ in each block is less than $N \cdot$ PRF,

After the subaperture division, the signal described by (5) is multiplied by a Doppler shift function $H_{2, n}\left(t_{a}, m\right)=$ $\exp \left(-j 2 \pi t_{a} f_{d c, i}\left(f_{c}\right)\right)$ to move the Doppler spectrum to the Doppler baseband. Then, the Doppler ambiguity can be resolved by spatial filtering [3-5] for each subband signal. The spatial filtering is a weighting operation of a multichannel signal for each subband in the Doppler domain. Considering the great amount of SF MIMO-SAR data, in order to reduce the computational burden of weight vectors of spatial filtering, the signals are multiplied by compensating functions to make the weight vectors independent of specific Doppler frequency [5], and the compensating functions can be constructed as

$$
H_{3, n}\left(f_{a}, m\right)=\exp \left(-j 2 \pi \frac{X_{n m}^{\prime}}{v}\left(f_{a}+f_{d c, i}\left(f_{c}\right)\right)\right),
$$

where $f_{a} \in(-N \cdot \mathrm{PRF} / 2,-(N-1) \cdot \mathrm{PRF} / 2)$ is the Doppler frequency. Then the weight vectors of the spatial filter can be obtained by

$$
\left[\mathbf{w}_{n}(1), \ldots, \mathbf{w}_{n}(m), \ldots, \mathbf{w}_{n}(N)\right]=\mathbf{A}_{n}^{-1},
$$

where

$$
\begin{gathered}
\mathbf{A}_{n}=\left[\mathbf{a}_{n}(1), \mathbf{a}_{n}(2), \ldots, \mathbf{a}_{n}(N)\right]^{T}, \\
a_{m}=\left[1, \exp \left(j 2 \pi \frac{X_{m}}{v} \mathrm{PRF}\right), \ldots,\right. \\
\left.\quad \exp \left(j 2 \pi \frac{X_{m}}{v}(N-1) \mathrm{PRF}\right)\right]^{T} .
\end{gathered}
$$

Then, the Doppler spectrum can be reconstructed by

$$
S_{2, n}\left(t, f_{a}+m \cdot \mathrm{PRF}\right)=\mathbf{S}_{1, n} \mathbf{w}_{n}(m),
$$

where $\mathbf{S}_{1, n}=\left[S_{1, n}\left(t, f_{a}, 1\right), \ldots, S_{1, n}\left(t, f_{a}, N\right)\right]$ is the multichannel signal vector of subband $n$. It can be observed from (9) that $\mathbf{a}_{n}(m)$ is independent of specific Doppler bin, and thus $\mathbf{w}_{n}(m)$ does not need to be updated with azimuth Doppler bins, which can sufficiently reduce the computation burden.

After the Doppler ambiguity resolving, the Doppler spectrum of subband $n$ signal can be expressed as

$$
\begin{aligned}
S_{2, n}( & \left.t, f_{d}\right) \\
= & w_{r}\left(t-\frac{2}{c} R_{\text {ref }}\left(f_{d}\right)\right) W_{\text {azi }}\left(f_{d}\right) \\
& \times \exp \left(-j 2 \pi\left(f_{d}+f_{d c, i}\left(f_{c}\right)\right) \frac{X}{v}\right) \\
& \times \exp \left(-j \frac{2 \pi}{v} R_{b} \sqrt{\left(\frac{2 v}{c} f_{n}\right)^{2}-\left(f_{d}+f_{d c, i}\left(f_{c}\right)\right)^{2}}\right),
\end{aligned}
$$

where $f_{d} \in(-N \cdot \mathrm{PRF} / 2, N \cdot \mathrm{PRF} / 2)$ denotes the Doppler frequency. From (11), we can see that each subband signal is equivalent to a single channel signal, and the geometry differences between different subband signals are removed. Then the bandwidth synthesis can be performed in Doppler domain.

\section{Improved FBS Method in Doppler Domain}

In general, there are two popular methods for the bandwidth synthetic of SAR signal: the synthetic the time-domain bandwidth synthesis (TBS) method and the FBS method. The TBS method is accurate, but the long duration of the synthetic pulse causes a high computation burden making it inefficient [6]. The FBS method is also accurate and more efficient, so it is widely used for bandwidth synthesis of SAR signal $[7,8]$.

The FBS method proposed in [7] transforms the subband signals to range frequency domain and then shifts the spectrum of subband $n$ signal by $\Delta f_{c, n}$ and performs coherent summation to obtain the whole bandwidth signal. However, considering the signals are discrete, the spectrum can only be shifted by an integer number of frequency bins in frequency domain. So this method is precise only if the residual fractional part of $\Delta f_{c, n}$ is zero. A solution to this problem is $N$ times upsampling and then multiplying the signal of subband $n$ by a frequency shift function in time domain to shift the spectrum by $\Delta f_{c, n}$ [8]. However, the processing of upsampling adds an extra FFT, $N$ times zeropadding in frequency domain, and an extra IFFT to the processing of bandwidth synthesis, which is inefficient with respect to both the use of data space and processing time.

To avoid signal upsampling in range, an improved FBS method in Doppler domain is proposed. Firstly, a slight shift of the range frequency spectrum is performed. The shift 


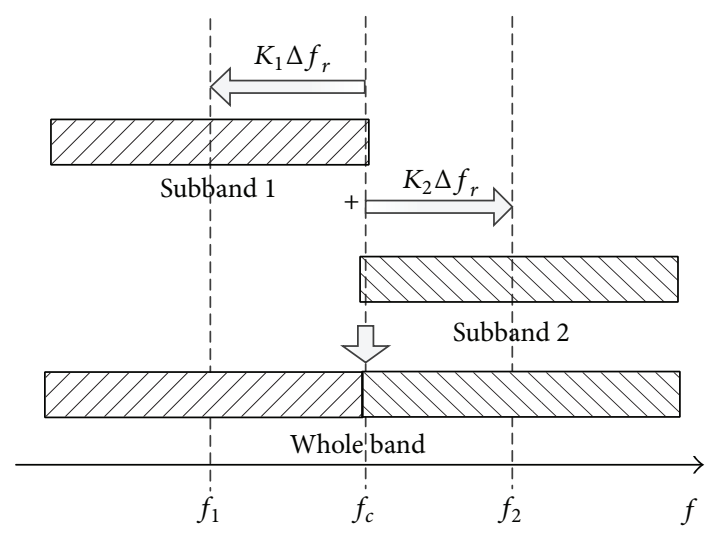

Figure 3: The spectrum shift and coherent summation $(N=2)$.

amount is the value of the fractional part of $\Delta f_{c, n}$, so the frequency shift functions can be constructed as

$$
H_{4, n}(t)=\exp \left[j 2 \pi t\left(\Delta f_{c, n}-K_{n} \Delta f_{r}\right)\right],
$$

where $\Delta f_{r}$ denotes the frequency interval of a frequency bin and $K_{n}$ is the integer part of $\Delta f_{c, n} / \Delta f_{r}$. Note that the frequency shifting amount is smaller than the value of a frequency bin, so that the range frequency spectrums do not alias.

Then, after the spectrum shift, the signals are transformed into range frequency domain. The center frequency distance between subband $n$ signal and the whole bandwidth signal spans over $K_{n}$ frequency bins, so spectrum shift and precise coherent summation can be performed as shown in Figure 3. Therefore, in comparison to the FBS method used in [8], the proposed improved FBS method can perform bandwidth synthesis precisely without upsampling of signal and thus lowers the computation load. After the coherent summation, the whole bandwidth signal in $2 \mathrm{D}$ frequency domain is obtained, which can be processed by conventional SAR imaging algorithms for corresponding modes $[4,11]$.

Figure 4 shows the flowchart of the proposed algorithm in the case of $N=2$. It consists of three parts: subaperture division, Doppler ambiguity resolving, and bandwidth synthesis.

Suppose $L_{r}$ and $L_{a}$ denote the range and azimuth sampling numbers of each subband signal, respectively. According to the flow of the proposed algorithm, the computational load of improved FBS method can be written as $(1 / 2) N L_{a} \log _{2} L_{r}+N L_{a} L_{r}$, while the computational load of FBS method presented in [8] is (1/2) NL $L_{a} \log _{2} L_{r}+$ $N L_{a} \log _{2} N L_{r}+N^{2} L_{a} L_{r}$. Therefore, the proposed improved FBS method can sufficiently lower the computational load of bandwidth synthesis.

\section{Simulation and Raw Data Results}

In this section, point targets simulation and constructed data processing results are carried out to verify the validity of the proposed algorithm.
TABLE 1: Main system parameters of point target simulation.

\begin{tabular}{lc}
\hline Number of Tx/Rx & 2 \\
Pulse duration & $10 \mu \mathrm{s}$ \\
Acquisition time & $6 \mathrm{~s}$ \\
Rotational distance & $800 \mathrm{~km}$ \\
PRF & $1598 \mathrm{~Hz}$ \\
Step frequency & $250 \mathrm{MHz}$ \\
Center frequency of subband 1 & $9.65 \mathrm{GHz}$ \\
Azimuth baseline & $2.3 \mathrm{~m}$ \\
Platform velocity & $7390 \mathrm{~m} / \mathrm{s}$ \\
Center distance of scene & $617 \mathrm{~km}$ \\
\hline
\end{tabular}

5.1. Simulation. The main parameters of a sliding spotlight SF MIMO-SAR system are given in Table 1 . A $3 \times 3$ point target array, of which the targets are uniformly distributed in a scene of $2 \mathrm{~km} \times 2 \mathrm{~km}$ in azimuth and range directions, is set in the illustrated scene.

After the subaperture division, the subband 1 signal in the $2 \mathrm{D}$ frequency domain before and after the spatial filtering is shown in Figures 5(a) and 5(b), respectively. It can be observed that the Doppler ambiguity is resolved by the spatial filtering, and the Doppler spectrum without aliasing is reconstructed for each subband signal. Then the improved FBS method is performed to combine the two subband signals, and the whole bandwidth signal in the 2D frequency domain is shown in Figure 6(a). Figure 6(b) shows the range images of a point target before and after bandwidth synthesis, from which one can see that the high range resolution is achieved after bandwidth synthesis. Then the imaging algorithm proposed in [11] is used to process the $2 \mathrm{D}$ reconstructed signal and the imaging result is obtained. The interpolated contour plots of three of the point targets are shown in Figure 7. These three point targets are denoted by $\mathrm{P}_{1}, \mathrm{P}_{2}$, and $\mathrm{P}_{3}$, respectively, and their $2 \mathrm{D}$ coordinates are $(-1 \mathrm{~km}, 616 \mathrm{~km}),(0 \mathrm{~km}, 617 \mathrm{~km})$, and $(1 \mathrm{~km}, 618 \mathrm{~km})$. The plots show that the point targets are well focused. The key image quality parameters, namely, the integrated sidelobe ratios (IRWs), the peak sidelobe ratios (PSLRs), and the integrated sidelobe ratios (ISLRs), are measured and shown in Table 2, which further verify the performance of the proposed algorithm.

5.2. Constructed Data Experiment. Since there are no SF MIMO-SAR data available for us, a single channel airborne Spotlight SAR data is used to construct SF MIMO-SAR data. The airborne Spotlight SAR data was collected on July, 2008, the main system parameters of which are given in Table 3 .

Firstly, the original data is transformed into range frequency domain and the whole range bandwidth is divided into two subbands, and then the subband 2 data is transformed into Doppler domain and multiplied by a slow time delay function to simulate an EPC difference from subband 1. Finally, the two subband data are downsampled by a factor of two into equivalent two channel undersampled data, respectively. Then a two-input two-output MIMO-SAR data in spotlight mode with two subbands is obtained. The main 
TABLE 2: Image quality of point targets.

\begin{tabular}{|c|c|c|c|c|c|c|}
\hline \multirow{2}{*}{ Target } & \multicolumn{3}{|c|}{ Range } & \multicolumn{3}{|c|}{ Azimuth } \\
\hline & IRW (m) & PSLR (dB) & $\operatorname{ISLR}(\mathrm{dB})$ & IRW (m) & PSLR (dB) & $\operatorname{ISLR}(\mathrm{dB})$ \\
\hline $\mathrm{P}_{1}$ & 0.255 & -13.29 & -10.01 & 0.280 & -13.39 & -10.48 \\
\hline $\mathrm{P}_{2}$ & 0.254 & -13.30 & -10.08 & 0.278 & -13.41 & -10.60 \\
\hline $\mathrm{P}_{3}$ & 0.255 & -13.30 & -10.03 & 0.281 & -13.41 & -10.51 \\
\hline
\end{tabular}

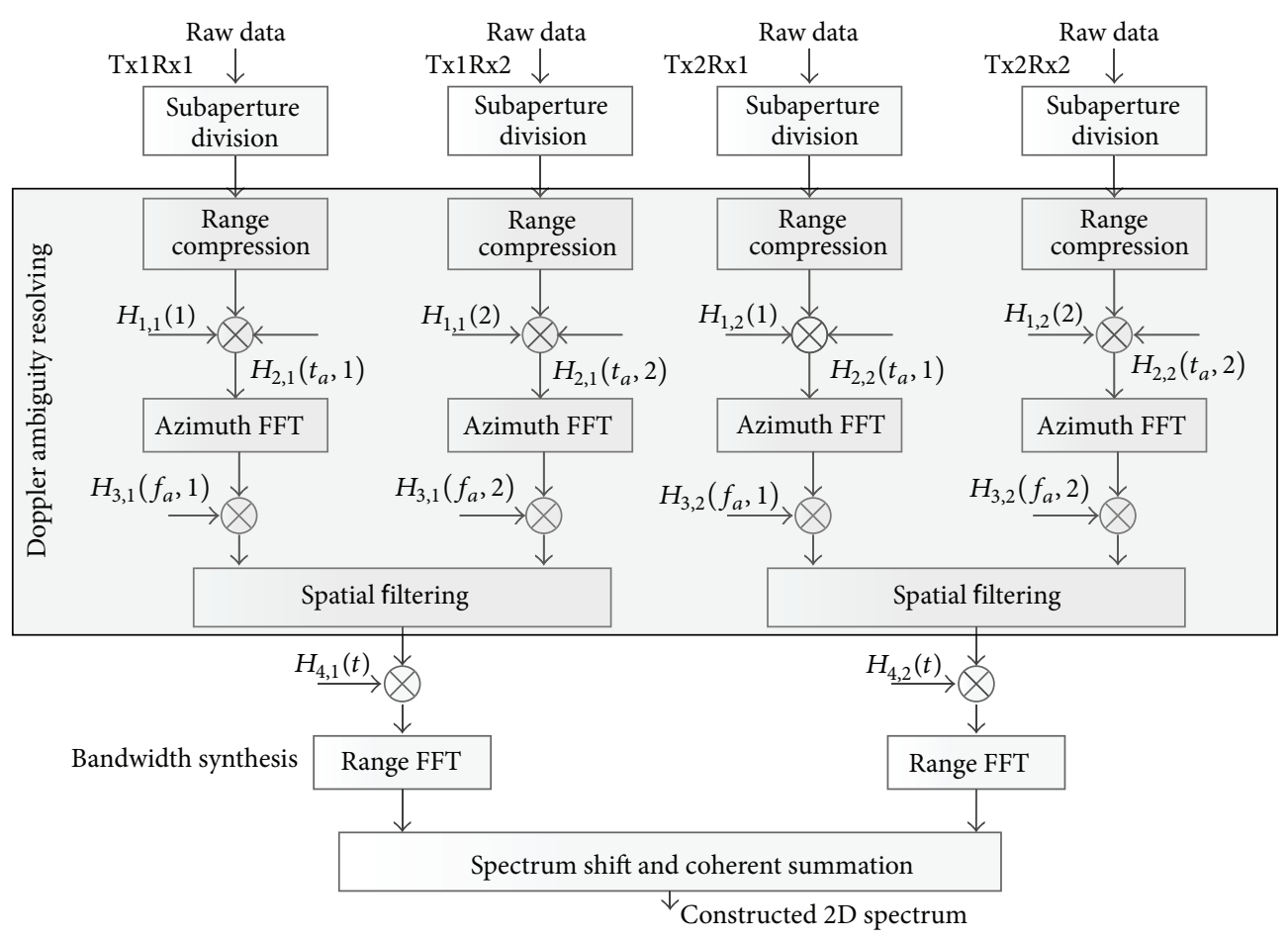

Figure 4: Flowchart of the proposed algorithm $(N=2)$.

TABLE 3: Main system parameters of original data.

\begin{tabular}{lc}
\hline Center frequency & $9.6 \mathrm{GHz}$ \\
Acquisition time & $10 \mathrm{~s}$ \\
PRF & $900 \mathrm{~Hz}$ \\
Signal bandwidth & $600 \mathrm{MHz}$ \\
Platform velocity & $97 \mathrm{~m} / \mathrm{s}$ \\
Center distance of scene & $20 \mathrm{~km}$ \\
\hline
\end{tabular}

parameters of the constructed SF MIMO-SAR data are given in Table 4.

Figure 8(a) shows the focused image of subband 1 data after Doppler ambiguity resolving, from which one can see that no ghost exists in azimuth. The focused image of the synthesized bandwidth signal is shown in Figure 8(b), and the comparison between the range images of a point target in the amplified area before and after bandwidth synthesis is shown in Figure 8(c), from which one can see that the range resolution is improved by the proposed improved FBS method. Therefore, the result of constructed real data processing also proves the effectiveness of the proposed algorithm.
TABLE 4: Main system parameters of constructed data.

\begin{tabular}{lc}
\hline Center frequency & $9.6 \mathrm{GHz}$ \\
Acquisition time & $10 \mathrm{~s}$ \\
PRF & $450 \mathrm{~Hz}$ \\
Center frequency of subband 1 & $9.45 \mathrm{GHz}$ \\
Step frequency & $300 \mathrm{MHz}$ \\
Platform velocity & $97 \mathrm{~m} / \mathrm{s}$ \\
Center distance of scene & $20 \mathrm{~km}$ \\
Azimuth baseline & $0.22 \mathrm{~m}$ \\
\hline
\end{tabular}

\section{Conclusion}

This paper presents an efficient algorithm to reconstruct SF MIMO-SAR signal in the spotlight and sliding spotlight modes. A Doppler ambiguity resolving method based on the subaperture division is presented to reconstruct the Doppler spectrum of each subband signal. Then, considering the high computation load of SF MIMO-SAR signal synthesis, an improved FBS method is proposed to avoid upsampling of data and thus lower the computation load of bandwidth synthesis. Results of point targets simulation and constructed 


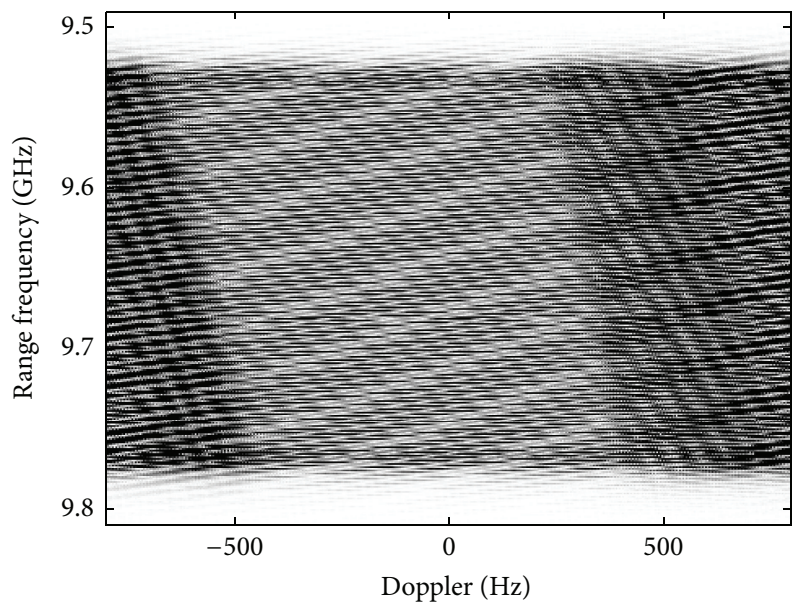

(a)

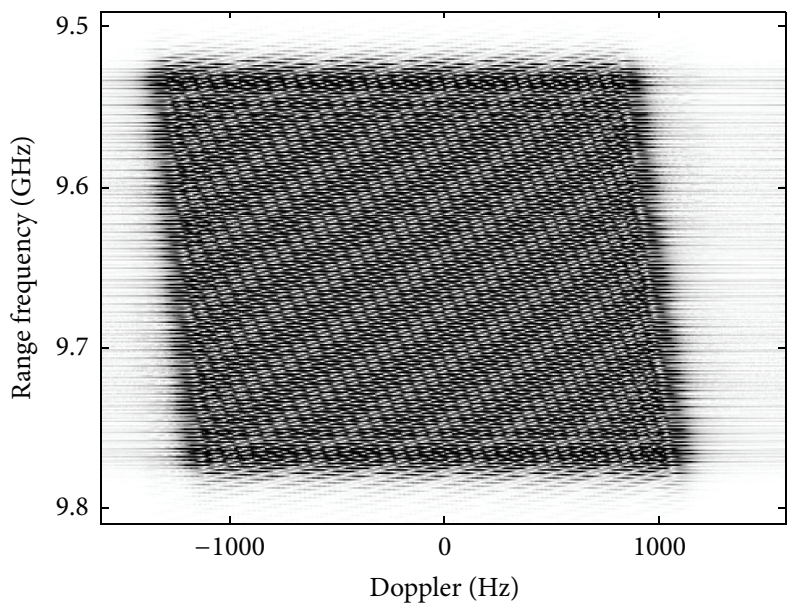

(b)

FIGURE 5: 2D spectrum (a) before and (b) after spatial filtering.

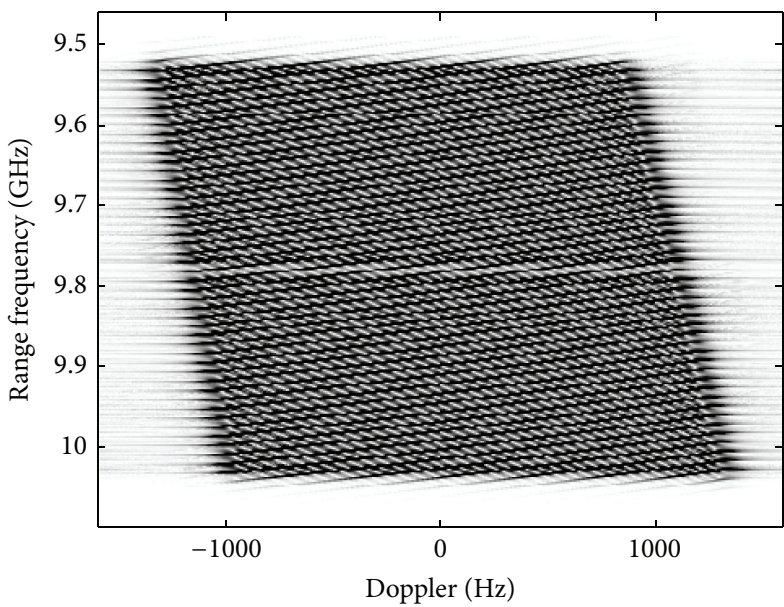

(a)

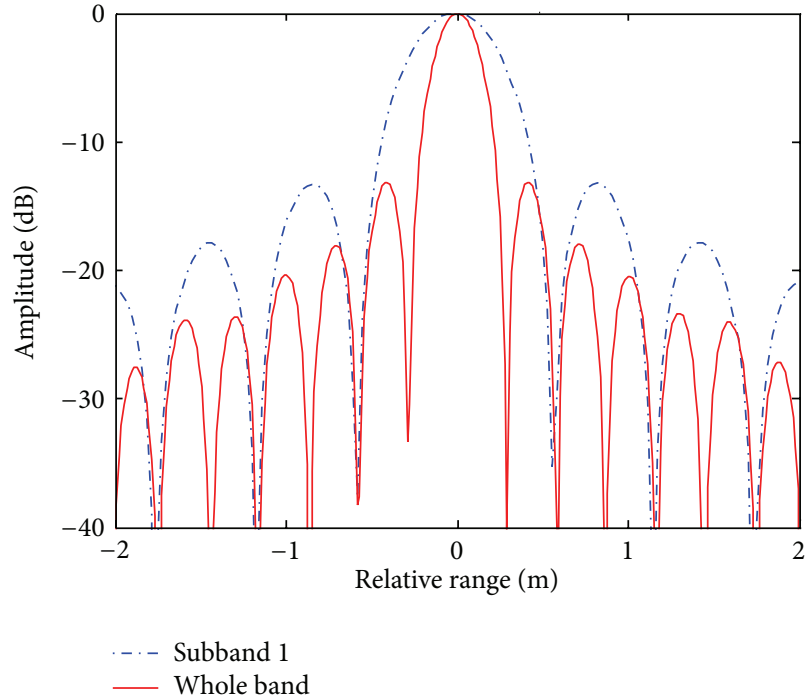

(b)

FIGURE 6: (a) 2D spectrum of the whole bandwidth signal. (b) Range images of a point target before and after bandwidth synthesis.

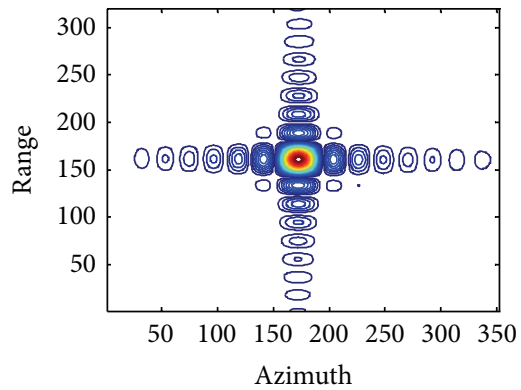

(a)

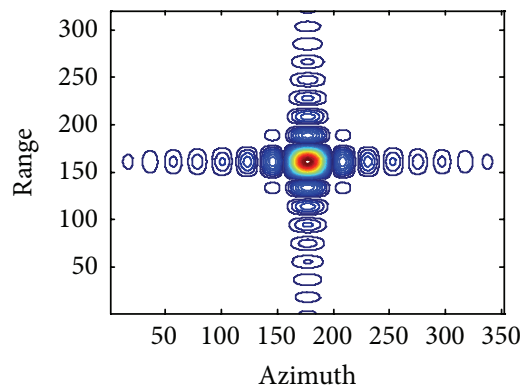

(b)

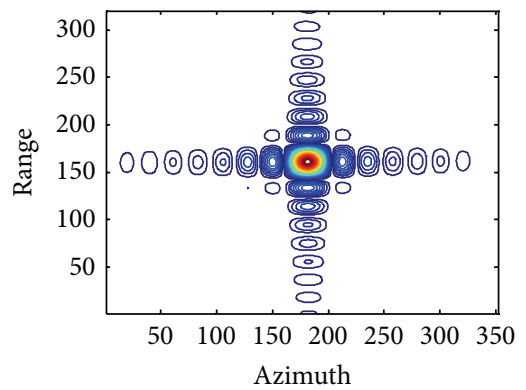

(c)

Figure 7: Contour plots of targets. 


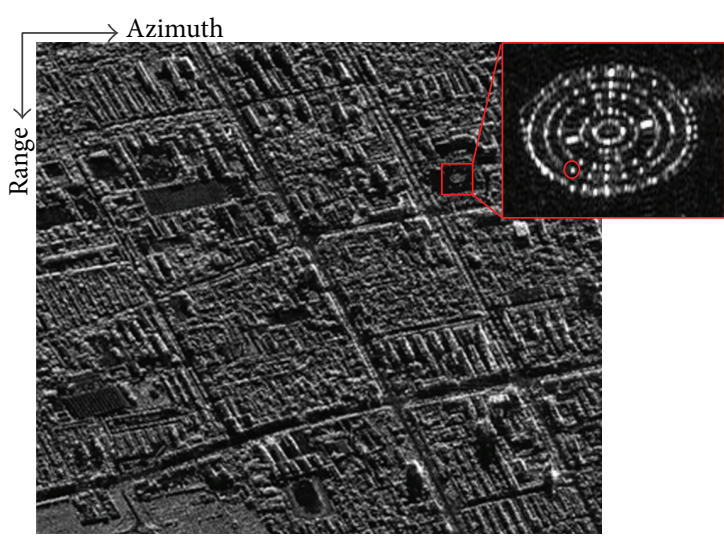

(a)

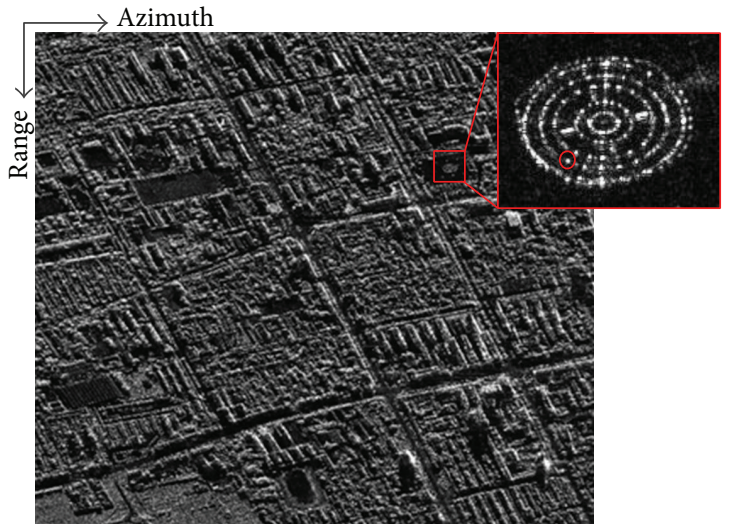

(b)

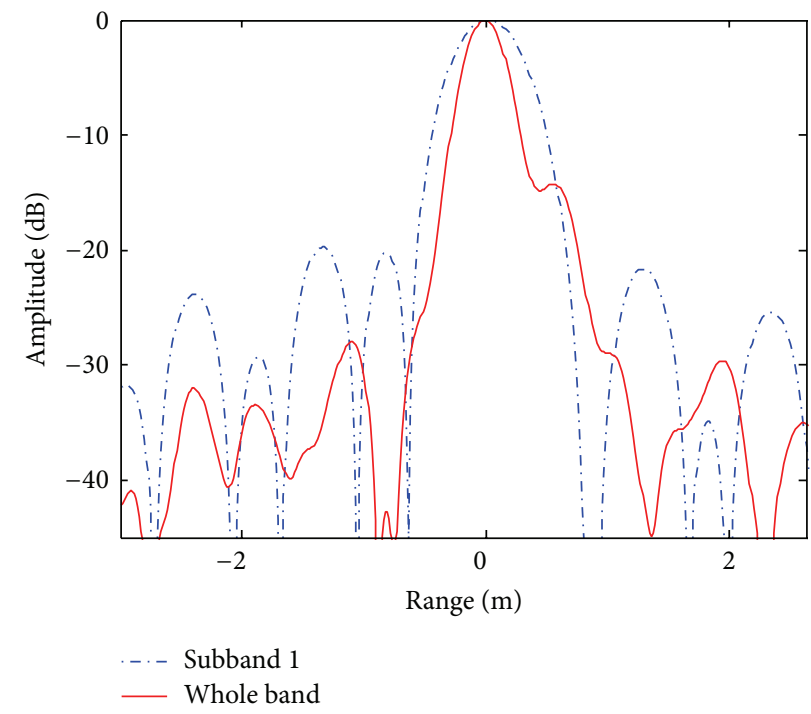

(c)

FIgURE 8: Results from an airborne spotlight SAR. (a) The image of subband 1 data after Doppler ambiguity resolving. (b) The image of the synthesized bandwidth data. (c) The range comparison of a point target in the amplified area.

data processing have validated the effectiveness of the proposed algorithm.

\section{Conflict of Interests}

The authors declare that there is no conflict of interests regarding the publication of this paper.

\section{Acknowledgment}

This work was supported by the National Natural Science Foundation of China under Grant 61301292.

\section{References}

[1] A. Freeman, W. T. K. Johnson, B. Huneycutt et al., "The myth of the minimum SAR antenna area constraint," IEEE Transactions on Geoscience and Remote Sensing, vol. 38, no. 1, pp. 320-324, 2000 .
[2] A. Currie and M. A. Brown, "Wide-swath SAR," IEE Proceedings F: Radar and Signal Processing, vol. 139, no. 2, pp. 122-135, 1992.

[3] N. Gebert, G. Krieger, and A. Moreira, "Multichannel azimuth processing in ScanSAR and TOPS mode operation," IEEE Transactions on Geoscience and Remote Sensing, vol. 48, no. 7, pp. 2994-3008, 2010.

[4] Q. Chen, Y. Deng, R. Wang, and Y. Liu, "Investigation of multichannel sliding spotlight SAR for ultrahigh-resolution and wide-swath imaging," IEEE Geoscience and Remote Sensing Letters, vol. 10, no. 6, pp. 1339-1343, 2013.

[5] G. Sun, M. Xing, X. Xia, Y. Wu, P. Huang, and Z. Bao, "Multichannel Full-aperture Azimuth Processing for beam steering SAR," IEEE Transactions on Geoscience and Remote Sensing, vol. 51, no. 9, pp. 4761-4778, 2013.

[6] P. Berens, "SAR with ultra-high range resolution using synthetic bandwidth," in Proceedings of the IEEE International Geoscience and Remote Sensing Symposium (IGARSS '99), vol. 3, pp. 1752 1754, Hamburg, Germany, July 1999.

[7] A. J. Wilkinson, R. T. Lord, and M. R. Inggs, "Stepped-frequency processing by reconstruction of target reflectivity spectrum," in 
Proceedings of the South African Symposium on Communications and Signal Processing (COMSIG '98), pp. 101-104, Rondebosch, South Africa, September 1998.

[8] Y. Deng, H. Zheng, R. Wang, J. Feng, and Y. Liu, "Internal calibration for stepped-frequency chirp SAR imaging," IEEE Geoscience and Remote Sensing Letters, vol. 8, no. 6, pp. 1105-1109, 2011.

[9] Q. Wu, M. Xing, Z. Bao, and H. Shi, "Wide swath, high range resolution imaging with MIMO-SAR," in IET International Radar Conference, April 2009.

[10] X. Luo, R. Wang, Y. Deng, and W. Xu, "Influences of channel errors and interference on the OFDM-MIMO SAR," in Proceedings of the IEEE Radar Conference, 2013.

[11] G. Sun, M. Xing, Y. Wang, Y. Wu, Y. Wu, and Z. Bao, "Sliding spotlight and TOPS SAR data processing without subaperture," IEEE Geoscience and Remote Sensing Letters, vol. 8, no. 6, pp. 1036-1040, 2011. 

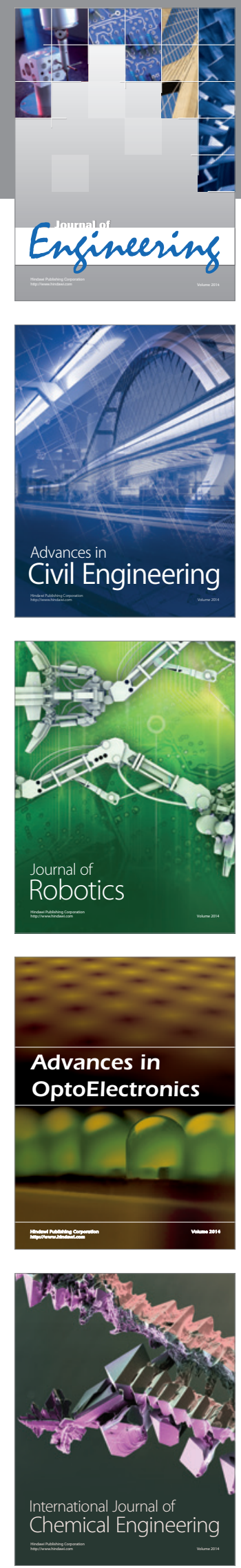

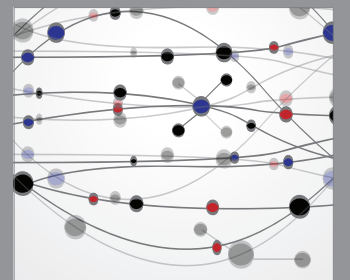

The Scientific World Journal
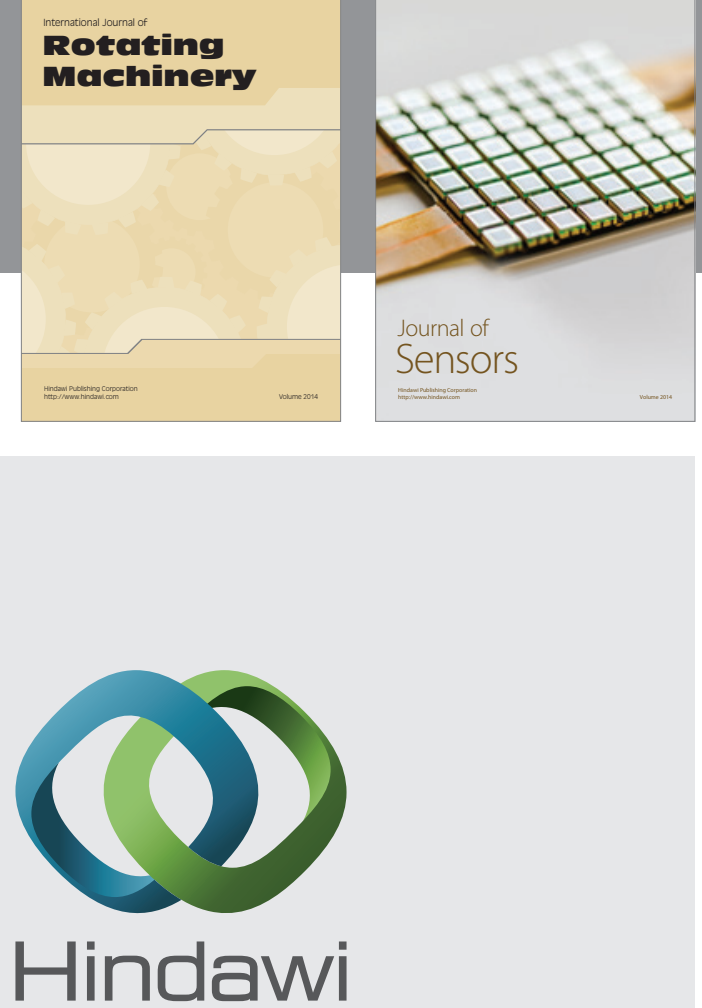

Submit your manuscripts at http://www.hindawi.com
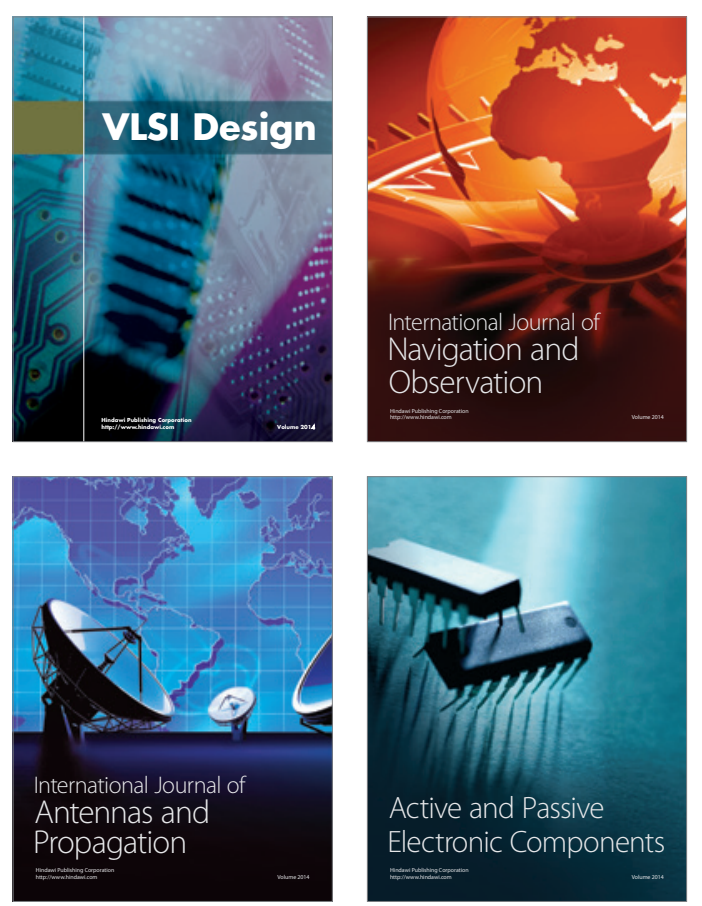
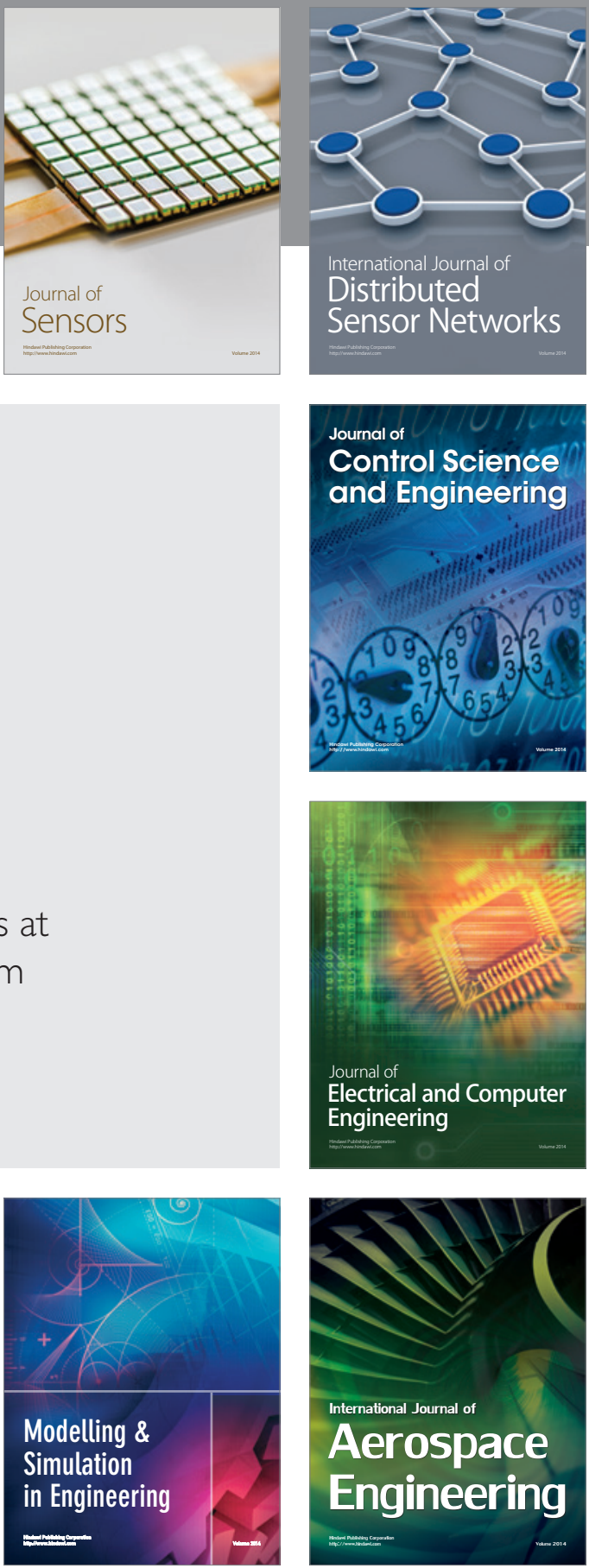

Journal of

Control Science

and Engineering
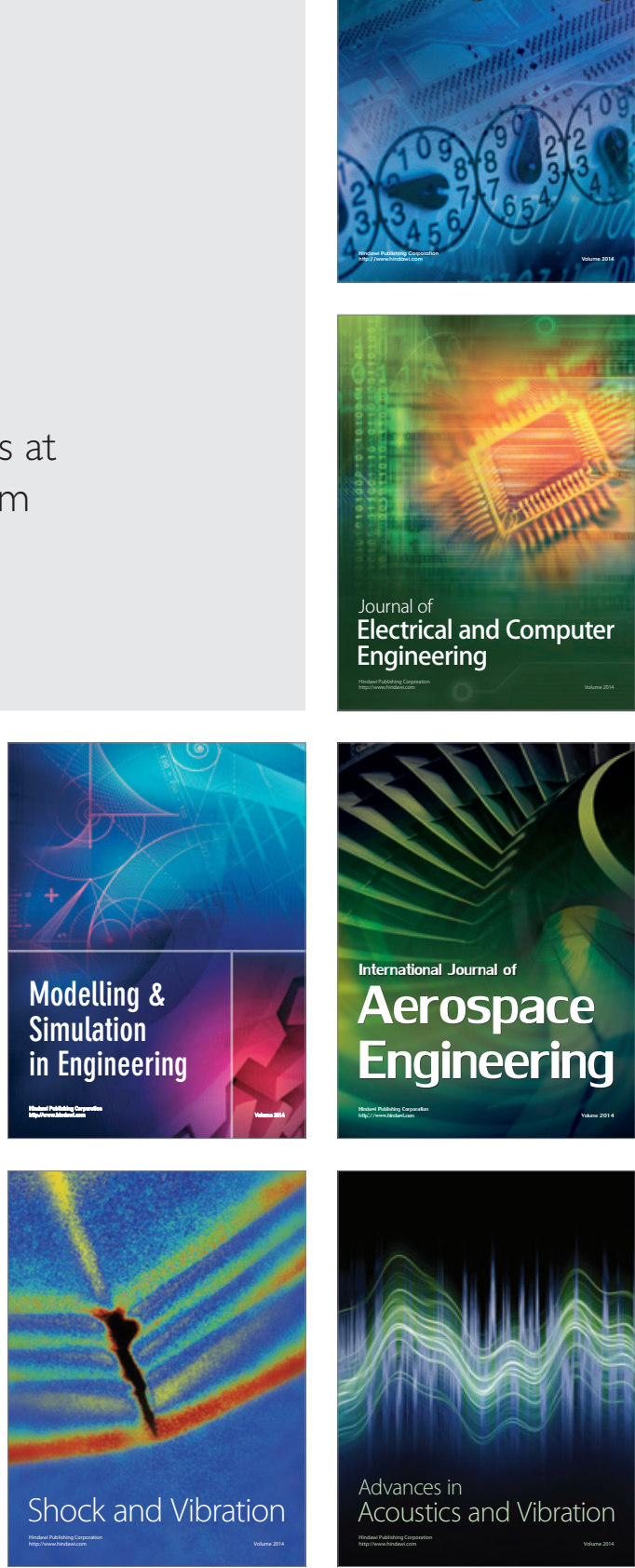